\title{
Designs of Coherent Optical Fast OFDM and Performance Comparison to Conventional OFDM
}

\author{
Jian Zhao ${ }^{1}$ and Andrew D. Ellis ${ }^{2}$ \\ ${ }^{I}$ Photonic Systems Group, Tyndall National Institute and University College Cork, Lee Maltings, Dyke Parade, Cork, Ireland \\ ${ }^{2}$ Aston Institute of Photonic Technology, Aston University, Birmingham B4 7ET, UK \\ Email: jian.zhao@tyndall.ie
}

\begin{abstract}
We discuss practical designs of coherent optical fast OFDM, and compare the performance of this scheme to conventional OFDM to identify its suitable application scenarios. OCIS codes: (060.2330) Fiber optics communications; (060.4080) Modulation
\end{abstract}

\section{Introduction}

Fast orthogonal frequency division multiplexing (F-OFDM), with subcarrier spacing reduced to half of that in conventional OFDM, was recently introduced to optical communications [1]. World-first experiments were demonstrated to verify its implementation feasibility at both $1.55-\mu \mathrm{m} \mathrm{[2]} \mathrm{and} \mathrm{2- \mu m} \mathrm{[3]} \mathrm{wavelengths.} \mathrm{This} \mathrm{technique}$ has been investigated using coherent [4-7], full-field [8], and direct [9-11] detections. In this paper, we will discuss the practical designs of coherent optical F-OFDM, and compare this technique to conventional OFDM to identify its suitable application scenarios.

\section{Experimental setup and principle}

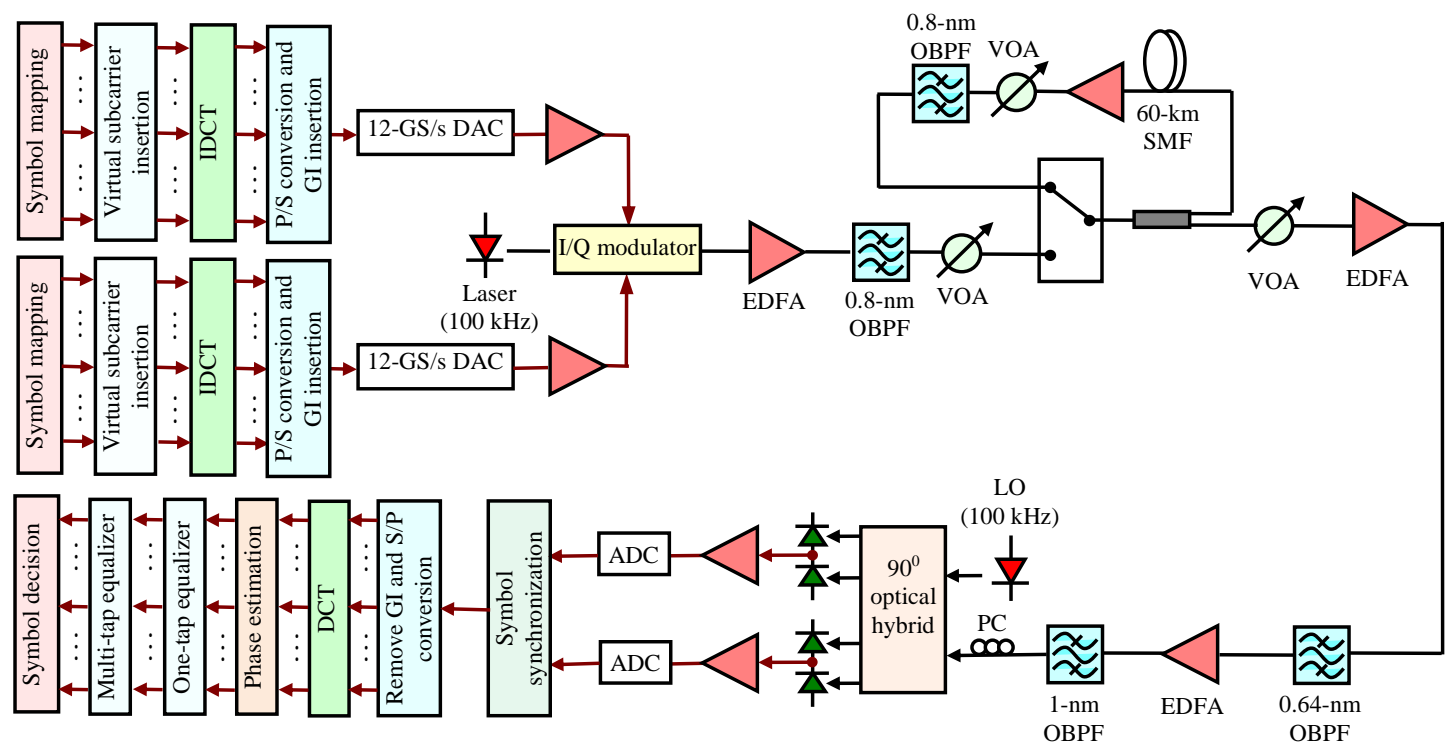

Fig. 1. Experimental setup of coherent 16QAM optical F-OFDM

Figure 1 illustrates the experimental setup. Two bi-polar four-amplitude-shift-keying (4-ASK) data were encoded with Gray coding in Matlab. Each F-OFDM frame consisted of a start-of-frame (SOF) symbol, training symbols (TSs) for channel estimation, and payload data. The SOF symbol was used to enable precise symbol synchronization at the receiver. The values of the subcarriers in the SOF symbol were set to be non-zero and real only for $a_{\mathrm{i}}$ with $i=$ $4 p$ (Fig. 2(a)), where $p$ is an integer. In the time domain, the SOF symbol was periodic and symmetric (Fig. 2(b)). Symbol synchronization based on this SOF was dispersion and format transparent [4]. In the TSs and payload, the inverse discrete cosine transform (IDCT) was used to multiplex the subcarriers with 128 points, of which 102 and 4 subcarriers were used for data transmission and phase estimation, respectively. The first two subcarriers were not modulated, allowing for AC-coupled amplifiers. The last 20 subcarriers were zero-padded to avoid aliasing. After IDCT and parallel-to-serial (P/S) conversion, 6 samples were added to each symbol as a guard interval (GI). Different from conventional OFDM which used the cyclic prefix as a GI, a symmetric extension based GI was required in optical F-OFDM to ensure the sub-channel orthogonality in the presence of chromatic dispersion (CD) (Fig. 2(c)) [5]. After GI insertion, the signal was downloaded to a 12-GS/s arbitrary waveform generator. 
A laser with 100-kHz linewidth was used to generate the optical carrier. The electrical F-OFDM signals were fed into the in-phase and quadrature arms of an optical I/Q modulator to generate an optical 16QAM F-OFDM signal. The input signals had a peak-to-peak driving swing of $0.5 V_{\pi}$. The generated optical signal was amplified by an erbium doped fiber amplifier (EDFA), filtered by a $0.8-\mathrm{nm}$ optical band-pass filter (OBPF), and transmitted over a re-circulating loop comprising 60-km single-mode fiber (SMF) with 15-dB fiber loss. The noise figure of the EDFA was $5 \mathrm{~dB}$ and another 0.8-nm OBPF was used in the loop to suppress the amplified spontaneous emission noise. The launch power per span was around $-5 \mathrm{dBm}$. At the receiver, the optical signal was detected with a pre-amplified coherent receiver and a variable optical attenuator (VOA) was used to vary the optical signal-to-noise ratio (OSNR) for the bit error rate (BER) measurements. The pre-amplifier was followed by an OBPF with a 3-dB bandwidth of $0.64 \mathrm{~nm}$, a second EDFA, and another OBPF with a 3-dB bandwidth of $1 \mathrm{~nm}$. A polarization controller (PC) was used to align the polarization of the filtered F-OFDM signal before entering the signal path of a $90^{\circ}$ optical hybrid. The optical outputs of the hybrid were connected to two balanced photodiodes with 40-GHz bandwidths, amplified by $40-\mathrm{GHz}$ electrical amplifiers, and captured using a 50-GS/s real-time oscilloscope. The receiver algorithms included interpolation, down-sampling, precise symbol synchronization, phase estimation, and one-tap equalizers for dispersion compensation. The coefficients of one-tap equalizers were estimated using the least-square (LS) based method [7]. When residual frequency offset (RFO) existed, multi-tap equalizers were used to mitigate the intercarrier interference (ICI) [6]. The total number of measured 16QAM symbols was around 240,000.

Subcarrier data of the SOF symbol

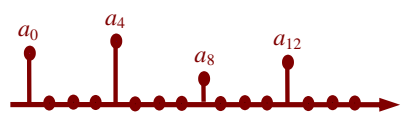

(a)
Time domain signal of the SOF symbol

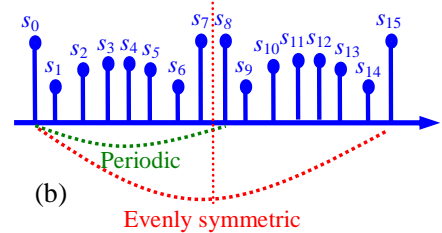

Time domain signal of the payload (including GI)

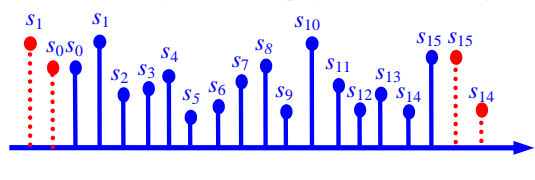

(c)
Symmetric extension based GI

Fig. 2. (a) Subcarrier allocation and (b) the time-domain signal of the SOF symbol; (c) symmetric extension based GI to preserve the sub-channel orthogonality in the presence of CD. (a)-(c): the subcarrier number is assumed to be 16.

\section{Results and discussions}
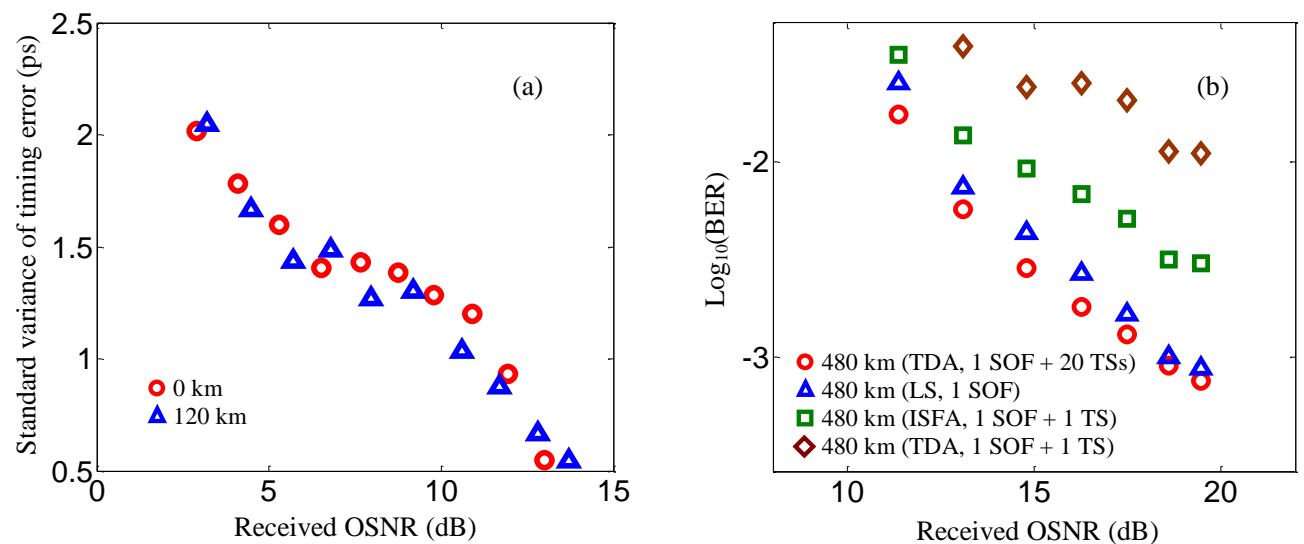

Fig. 3. (a) The standard variance of the timing error versus the received OSNR at 0 and $120 \mathrm{~km}$. (b) BER versus the received OSNR at $480 \mathrm{~km}$. The subcarrier number for averaging in intra-symbol frequency-domain averaging (ISFA) is 5. (a)-(b): The amplitude of data subcarriers in the SOF is twice that of the payload to maintain the same average power. The RFO is zero and the subcarrier number is 128.

Fig. 3(a) shows the standard variance of the timing error using the investigated symbol synchronization method. It can be seen that the standard variance was less than 1 ps for OSNR values larger than $12 \mathrm{~dB}$, and was still less than $2 \mathrm{ps}$ even when the OSNR was reduce to $3 \mathrm{~dB}$ after either 0 - or 120-km transmission. Note that the SOF symbol is inserted before the TSs and payload, so is also transparent to the formats used in the payload. Fig. 3(b) shows BER versus the received OSNR at $480 \mathrm{~km}$ using different methods to estimate the one-tap equalizer's coefficients: conventional time-domain averaging (TDA), intra-symbol frequency domain averaging (ISFA), and the LS method. It can be seen that when the number of TSs was one, the performance was significantly degraded when TDA was used, due to high noise levels. ISFA improved the performance but still exhibited a large penalty. In contrast, joint symbol synchronization and dispersion estimation could be achieved by the LS method using single SOF symbol. 
The RFO would introduce ICI, which can be mitigated by frequency-domain multi-tap equalizers. Fig. 4(a) and (b) compare the ICI coefficients, $h_{\mathrm{k}}$, for F-OFDM and conventional OFDM. It can be seen that $\mid$ real $\left\{h_{\mathrm{k}}\right\} \mid$ in FOFDM are almost zero for $k \neq 0$ whilst those in conventional OFDM have much higher values. The ICI in F-OFDM is dominated by imag $\left\{h_{-1}\right\}$ and imag $\left\{h_{1}\right\}$. Fig. 4(c) shows BER versus the RFO at 18.5-dB OSNR using seven-tap equalizers and the insets show the constellation diagrams at $15-\mathrm{MHz}$ RFO. It can be seen that the BER of the conventional OFDM increased quickly as the RFO increased, and the RFO tolerance range at a BER of $10^{-3}$ was $\sim 5$ MHz. In contrast, F-OFDM exhibited a RFO tolerance more than four times greater than that of the conventional OFDM. Fig. 4(d) shows that as expected, a three-tap equalizer could compensate most of the ICI, resulting in significant performance improvement over a one-tap equalizer. Increasing the tap number to five could further improve the performance. As $h_{\mathrm{k}}$ was nearly zero for $|k|>2$, performance became stable for seven-tap equalizers.
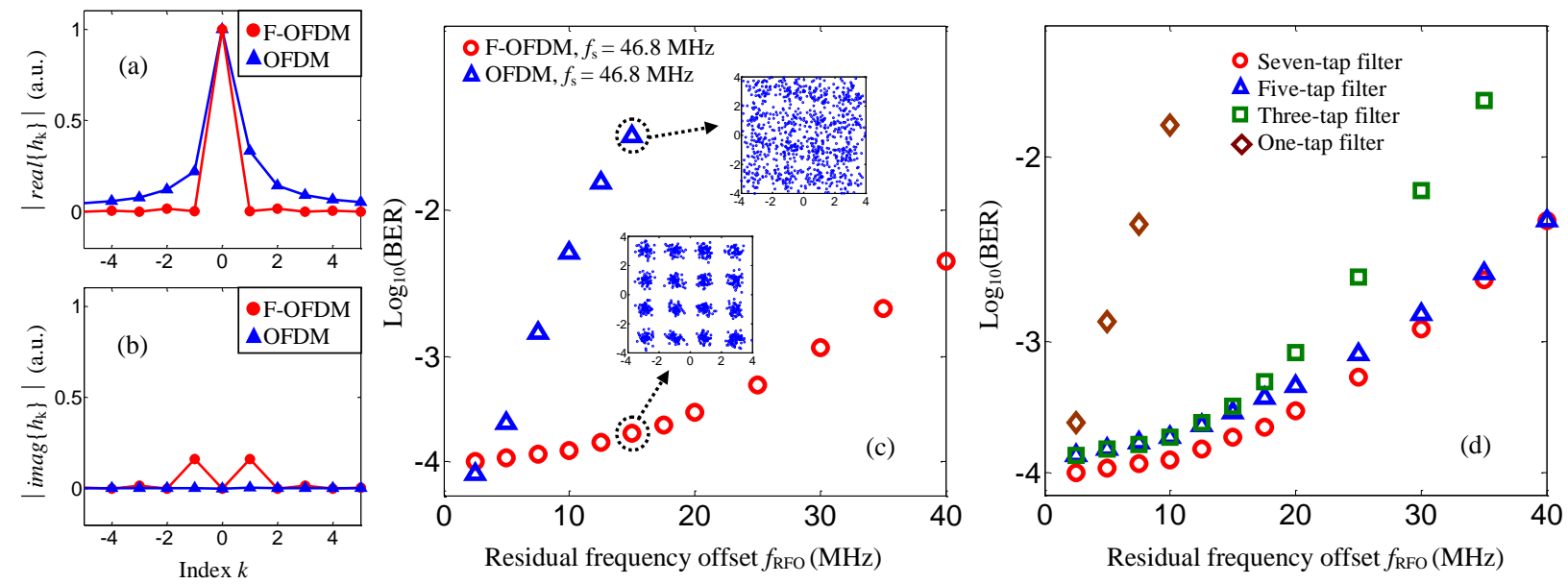

Fig. 4. Absolute amplitude of (a) real and (b) imaginary parts of the ICI coefficient $h_{\mathrm{k}}$ in the presence of residual frequency offset (RFO). The $\mathrm{RFO}, f_{\mathrm{RFO}}$, and the subcarrier spacing, $f_{\mathrm{s}}$, are 12 and $46.8 \mathrm{MHz}$, respectively; (c) BER versus $f_{\mathrm{RFO}}$ at 18.5 -dB OSNR using seven-tap FIR filter. Insets show the constellation diagrams of F-OFDM and conventional OFDM when $f_{\text {RFO }}=15$ MHz. (d) BER versus $f_{\text {RFO }}$ for optical F-OFDM using multi-tap equalizers with different tap numbers at 18.5-dB OSNR and 46.8-MHz $f_{\mathrm{s}}$.

\section{Conclusions}

We have discussed the implementation designs for coherent optical F-OFDM, and in particular, investigated the fast least-square based dispersion estimation in optical F-OFDM to achieve joint symbol synchronization and channel estimation using single SOF symbol with negligible performance penalty. We have also shown that optical F-OFDM exhibits a tolerance of RFO to subcarrier spacing ratio more than four times greater than that of conventional OFDM. This makes optical F-OFDM a promising technique for the future packet optical networks with fast tunable transceivers. This work was supported by Science Foundation Ireland under grant number 11/SIRG/I2124 and 06/IN/I969, and the EU $7^{\text {th }}$ Framework Program under grant agreement 318415 (FOX-C).

\section{References:}

[1] J. Zhao and A.D. Ellis, "A novel optical fast OFDM with reduced channel spacing equal to half of the symbol rate per carrier," IEEE/OSA Optical Fiber Communication Conference (2010), paper OMR1.

[2] S.K. Ibrahim, J. Zhao, D. Rafique, J. O’Dowd, and A.D. Ellis “Demonstration of world-first experimental optical fast OFDM system at 7.174Gbit/s and 14.348Gbit/s," European Conference on Optical Communication (2010), post-deadline paper PDP3.4.

[3] N. MacSuibhne et al, "Wavelength division multiplexing at $2 \mu \mathrm{m}$," European Conference on Optical Communication (2012), PDP Th.3.A.3.

[4] J. Zhao, S.K. Ibrahim, D. Rafique, P. Gunning, and A.D. Ellis, "Symbol synchronization exploiting the symmetric property in optical fast OFDM,” IEEE Photon. Technol. Lett. 23, 594-596 (2011).

[5] J. Zhao and A.D. Ellis, "Transmission of 4-ASK optical fast OFDM with chromatic dispersion compensation,” IEEE Photon. Technol. Lett. 24, 34-36 (2012).

[6] J. Zhao and A.D. Ellis, "Advantage of optical fast OFDM over OFDM in residual frequency offset compensation," IEEE Photon. Technol. Lett. 24, 2284-2287 (2012).

[7] J. Zhao and H. Shams, "Fast dispersion estimation in coherent optical 16QAM fast OFDM systems," Opt. Express 21, $2500-2505$ (2013).

[8] J. Zhao, "Intensity modulation full-field detection optical fast OFDM," IEEE J. Opt. Commun. Netw. 5, 465-474 (2013)

[9] E. Giacoumidis et al., "Experimental demonstration of cost-effective intensity modulation and direct detection optical fast OFDM over 40km SMF transmission," IEEE/OSA Optical Fiber Communication Conference (2012), paper JW2A.

[10] E. Giacoumidis et al., "Extensive comparison of optical fast OFDM and conventional OFDM for local and access networks," IEEE J. Opt. Commun. Netw. 4, 724-733 (2012).

[11] E. Giacoumidis, S. K. Ibrahim, J. Zhao, J. M. Tang, A. D. Ellis, and I. Tomkos, "Experimental and Theoretical Investigations of IntensityModulation and Direct-Detection Optical Fast-OFDM over MMF-links," IEEE Photon. Technol. Lett. 24, 52-54 (2012). 